\title{
Expansión de la atención integrada a las enfermedades prevalentes de la infancia en el Perú y su asociación con indicadores de salud *
}

\author{
Luis Huicho ${ }^{1}$, Miguel Dávila ${ }^{2}$, Fernando Gonzales ${ }^{2}$, Christopher Drasbek ${ }^{3}$, \\ Jennifer Bryce ${ }^{4}$, Cesar G. Victora ${ }^{5}$
}

Resumen

Objetivos: 1) Documentar las tendencias en la implementación de la atención integrada a las enfermedades prevalentes de la infancia (Aiepi) en los 24 departamentos del país, de 1996 al 2000. 2) Documentar las tendencias en los indicadores de cobertura de servicios de salud y en los de impacto (mortalidad y estado nutricional) para el mismo período. 3) Correlacionar los cambios en estos dos grupos de indicadores. $Y, 4)$ intentar descartar factores contextuales que puedan afectar las tendencias y las correlaciones observadas. Materiales y Métodos: Se realizó un análisis ecológico en el que las unidades de estudio fueron los 24 departamentos. Resultados: Para el 2000, 10,2\% de trabajadores clínicos (médicos y enfermeras) fueron capacitados en Aiepi, pero solo algunos departamentos mostraron tasas considerablemente mayores. No hubo asociaciones significativas entre la cobertura de capacitación clínica en Aiepi y los indicadores de utilización de consultas externas, cobertura de vacunas, mortalidad o desnutrición. La falta de asociación persistió luego de haber realizado el ajuste de varios factores contextuales incluyendo indicadores socioeconómicos y ambientales y la presencia de otros proyectos de salud del niño. Los agentes comunitarios de salud también fueron capacitados en Aiepi y la cobertura de capacitación no estuvo asociada con ninguno de los indicadores de proceso o de impacto, excepto una correlación positiva y significativa con el promedio de talla para edad. De acuerdo al modelo de impacto de la Evaluación Multi-País del Impacto, Costo y Efectividad de Aiepi (EMP), la implementación de Aiepi debe ser lo suficientemente fuerte para llevar a un impacto en la salud y la nutrición. Conclusiones: El soporte de los sistemas de salud para la implementación de Aiepi en el Perú estuvo lejos de ser adecuada, y esto, así como coberturas de capacitación relativamente bajas, pueden explicar porqué no se documentó el impacto esperado. Sin embargo, incluso los departamentos con altos niveles de cobertura de capacitación no mostraron un impacto. Se discute estos resultados en el contexto de otros dos estudios de la EMP, realizados en Tanzania y Bangladesh, en los que se constató un impacto positivo de Aiepi fuertemente implementado, y se propone posibles explicaciones para estas discrepancias.

Palabras clave Atención integral de la salud; salud infantil; impactos en la salud; Perú, salud pública.

* Versión traducida de: Luis Huicho, Miguel Dávila, Fernando Gonzales, Christopher Drasbek, Jennifer Bryce, Cesar G. Victora. Implementation of the integrated management of childhood illness (IMCl) strategy in Peru and its association with health indicators: an ecological analysis. Health Policy and Planning. 2005;20 Suppl 1:i32-i41.

1 Instituto de Salud del Niño y Universidad Nacional Mayor de San Marcos. Lima, Perú.

Organización Panamericana de la Salud. Lima, Perú.

3 Organización Panamericana de la Salud. Washington, USA.

42081 Danby Road, Ithaca, NY 14850 USA.

5 Universidad Federal de Pelotas, Brasil.
Implementation of the integrated management of childhood illnesses strategy in Peru and its association with health indicators: an ecological analysis

Abstract

Objectives: (1) To document trends in integrated management of childhood illnesses (IMCI) implementation in the 24 departments of Peru from 1996 to 2000. (2) To document trends in indicators of health services coverage and impact (mortality and nutritional status) for the same period. (3) To correlate changes in these two sets of indicators. And (4) to attempt to rule out contextual factors 
that may affect the observed trends and correlations. Materials and Methods: An ecological analysis was performed in which the units of study were the 24 departments. Results: By 2000, 10,2\% of clinical health workers were trained in IMCI, but some districts showed considerably higher rates. There were no significant associations between clinical IMCI training coverage and indicators of outpatient utilization, vaccine coverage, mortality or malnutrition. The lack of association persisted after adjustment for several contextual factors including socioeconomic and environmental indicators and the presence of other child health projects. Community health workers were also trained in IMCI, and training coverage was not associated with any of the process or impact indicators, except for a significant positive correlation with mean height for age. According to the MCE impactmodel, IMCI implementation must be sufficiently strong to lead to an impact on health and nutrition. Conclusions: Health systems supportfor IMCI implementation in Peru was far from adequate. This finding along with low training coverage level and a relatively low child mortality rate may explain why the expected impact was not documented. Nevertheless, even districts with high levels of training coverage failed to show an impact. Further national effectiveness studies of IMCI and other child interventions are warranted as these interventions are scaled up.

Keywords: Comprehensive health care; child health; impacts of health; Peru, public health.

\section{INTRODUCCIÓN}

Los niños menores de cinco años constituyen el $30 \%$ de la carga global de enfermedad en los países en desarrollo. Hasta $99 \%$ de las muertes en los niños ocurrió en estos países en 1999. La mayor parte de esas muertes se debe a pocas enfermedades comunicables y a desnutrición ${ }^{1}$ ). Los programas verticales que estuvieron dirigidos a controlar enfermedades específicas -tales como diarrea o neumoníaprobaron ser efectivos en los 1970 y los 1980. Sin embargo, como los principales problemas de salud del niño coexisten frecuentemente, se desarrolló en los 1990 la estrategia de Atención Integrada a las Enfermedades Prevalentes de la Infancia (Aiepi), para enfrentar la condición global del niño, considerando los diferentes factores que pudieran estar contribuyendo a la enfermedad. Esta estrategia está siendo implementada en más de 100 países, con el objetivo de reducir aún más las muertes de los niños por neumonía, diarrea, sarampión, malaria y desnutrición $\left(^{2}\right)$.

Aiepi comprende varias intervenciones curativas (por ejemplo, antibióticos, rehidratación oral, antimaláricos) y preventivas (por ejemplo, vacunas, micronutrientes, promoción de la lactancia maternal) bien conocidas. La eficacia de cada intervención de salud del niño incluida en la estrategia Aiepi ha sido ya confirmada por varios estudios $\left({ }^{3}\right)$, pero todavía queda por demostrar la efectividad de Aiepi en diferentes lugares, como una estrategia global. En 1998, se lanzó la iniciativa de Evaluación Multi-País del Impacto, Costo y Efectividad de Aiepi (EMP), con estudios en cinco países: Bangladesh, Brasil, Perú, Tanzania y Uganda $\left({ }^{4}\right)$. Fueron seleccionados diferentes países para brindar una amplia variabilidad de factores contextuales, incluyendo diferentes sistemas de salud, niveles y perfiles de mortalidad, características sociales y económicas.

El equipo de la EMP desarrolló un modelo de impacto que describe cómo se esperaba que la introducción de Aiepi redujera la mortalidad del niño y mejorara su estado nutricional. Se muestra una versión simplificada del modelo en la Figura $1\left({ }^{4}\right)$. La figura indica los tres componentes de Aiepi: las mejoras en los sistemas de salud, la capacitación de los trabajadores de salud y las intervenciones a nivel de la comunidad. Se esperaba que, si estos componentes eran implementados adecuadamente, resultarían en una búsqueda apropiada de atención de salud y en un incremento de la utilización de los servicios. Ésta, a su vez, resultaría en un mejor manejo de los episodios de enfermedad y en una mayor cobertura de intervenciones preventivas y, finalmente, en una mejora en la supervivencia y el estado nutricional.

El Perú fue incluido en la EMP por dos razones principales: era el país líder en las Américas en términos de la implementación de Aiepi y se disponía de datos secundarios de calidad aceptable sobre muchos de los 


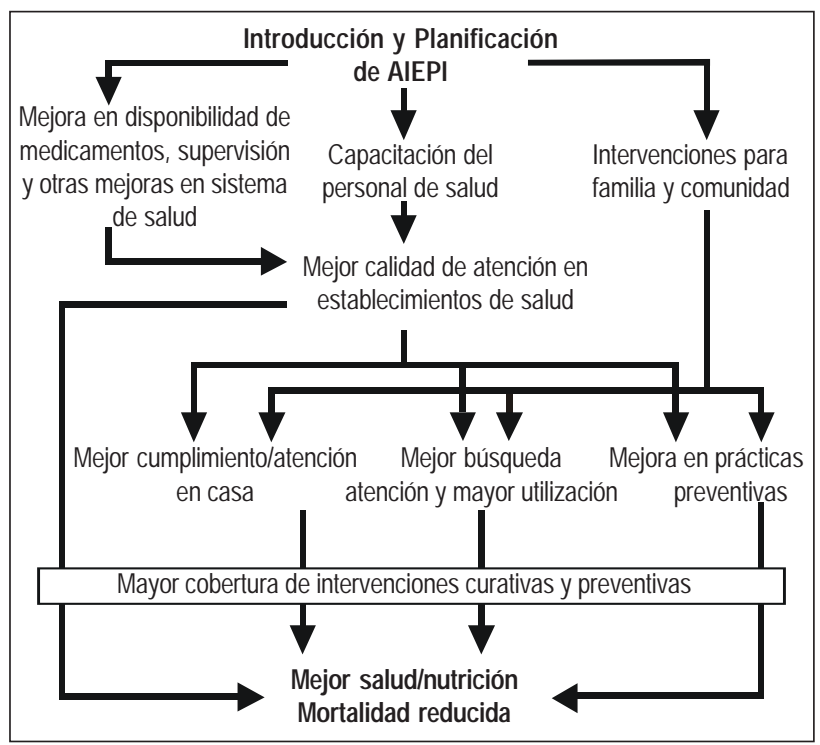

Figura 1. Modelo propuesto de impacto de Aiepi.

indicadores incluidos en el modelo de impacto (Figura 1). Además, brindaba una oportunidad para evaluar Aiepi en un país con mortalidad relativamente baja y con un sistema de salud bien estructurado, en contraste con los otros países de la EMP, en los que las tasas de mortalidad del niño eran altas y los sistemas de salud débiles.

Los objetivos de la EMP en el Perú incluyeron la descripción de la implementación de Aiepi en los 24 departamentos del país, desde 1996. Estos hallazgos fueron descritos recientemente $\left({ }^{5}\right)$. De acuerdo a la base de datos de la Organización Panamericana de la Salud (OPS), el número de cursos de capacitación incrementó hasta 1999, pero disminuyó posteriormente. Para el 2000, aproximadamente $10 \%$ de todos los médicos y enfermeras que trabajaban en el Ministerio de Salud (Minsa) había sido capacitado en Aiepi. La capacitación de los agentes comunitarios de salud, por otra parte, empezó en 1997 y aumentó significativamente de 1999 en adelante. Sin embargo, las Disas en las que la capacitación clínica fue más intensa no fueron las mismas en las que la capacitación en Aiepi comunitario fue más fuerte. Nuestra evaluación también encontró que Aiepi estaba insuficientemente institucionalizada -tanto a nivel nacional como de las Disas-, y que la supervisión de los trabajadores capacitados era infrecuente. Sin embargo, la provisión de medicamentos, vacunas y equipos pareció ser adecuada, en general.

Además de los indicadores de implementación, nuestro estudio también recogió datos sobre tendencias temporales en los indicadores de utilización y cobertura de servicios de salud y en los indicadores de impacto (mortalidad y estado nutricional). En el presente artículo examinamos si la implementación de Aiepi estaba positivamente asociada con cambios en estos indicadores, analizando las tendencias en los 24 departmentos del país. La pregunta que planteamos fue si Aiepi, implementada bajo condiciones rutinarias a nivel nacional, tenía un impacto mensurable sobre la supervivencia y el estado nutricional de los niños.

\section{MATERIALES Y MÉTODOS}

Se realizó un estudio ecológico que combinó la investigación de información geográfica e histórica para las 34 regiones de salud (Disas) y para los 24 departmentos (divisiones políticas) del Perú. El estudio comprendió el período de 1996 al 2000.

Los datos fueron obtenidos de fuentes primarias y secundarias. Los indicadores relacionados con la implementación de Aiepi (cobertura de capacitación, provisión de medicamentos, vacunas y equipos, supervisión, referencia, actividades comunitarias, planificación y presupuestación) fueron recolectados a través de cuestionarios estandarizados respondidos por el personal de cada Disa durante una visita de 3 a 4 días por un entrevistador capacitado. Estos datos fueron revisados para verificar su consistencia y contrastados contra información disponible en el Ministerio de Salud y la oficina nacional de la OPS. Los indicadores de utilización de servicios de salud también fueron obtenidos de estas fuentes. Cuando se detectó inconsistencias, se realizó contactos adicionales con los coordinadores de las Disas para completar o corregir la información. 
Los datos de implementación obtenidos de las 34 Disas fueron agregados en 24 departamentos para hacerlos comparables con la base geográfica de los datos secundarios. Para el proceso de consolidación, se unió las siguientes Disas, de acuerdo a la división política departamental del Perú: Chota, Cutervo y Jaén fueron incorporados a Cajamarca; Lima Sur, Lima Norte, Lima Centro, Lima Este y Callao fueron incorporados a Lima; Bagua fue incorporada a Amazonas, Andahuaylas a Apurímac, y Sullana a Piura. Para la consolidación, se ponderó los resultados por tamaño poblacional. Luego, se unió ambas bases de datos en una sola.

Se utilizó las siguientes variables de implementación en los análisis realizados:

- Capacitación en Aiepi clínico: el porcentaje de médicos y enfermeras empleados por el MINSA en cada departamento que fueron capacitados en el curso de Aiepi clínico entre 1996 y el 2000.

- Capacitación en Aiepi comunitario: razón de agentes comunitarios de salud (ACS) capacitados en el curso de Aiepi comunitario, por 10000 niños menores de cinco años, 1996-2000. Puesto que los ACS incluyó a aquellos pertenecientes a organizaciones gubernamentales y no gubernamentales, no se pudo obtener estimados confiables del número total $\mathrm{y}$, por tanto, el indicador es expresado por 10000 niños y no como porcentaje de todos los ACS.

Se utilizó fuentes de datos adicionales. El Instituto Nacional de Estadística e Informática (Inei) es la oficina estadística oficial en el país, y está a cargo de los censos demográficos (el último de los cuales se realizó en 1993) y de las encuestas nacionales de hogares (Enaho) llevadas a cabo anualmente entre 1996 y el $2000\left(^{6}\right)$. Las Encuestas Nacionales Demográficas y de Salud Familiar (Endes) fueron llevadas a cabo en 1996 y el 2000 por el Inei. Los datos de Endes son representativos a nivel departamental para la mayoría de indicadores $\left({ }^{7,8}\right)$. La oficina de Estadística e Informática del Ministerio de Salud compila las estadísticas anuales sobre provisión y utilización de servicios de salud y vigilancia de morbilidad, en base a los informes de los establecimientos de salud. Tambén produce datos de mortalidad en base a los registros de estadísticas vitales $\left({ }^{9}\right)$.

Estas fuentes brindaron información sobre:

- Utilización de consultas externas: promedio del número anual de atenciones de niños menores de cinco años, 2000 (OGEI/Minsa).

- Cobertura de vacunas: porcentaje de niños de 18 a 29 meses de edad que recibieron todas las vacunas básicas, confirmado por el carné de vacunaciones, 2000 (Endes).

El impacto de Aiepi fue evaluado a través de indicadores de nutrición y mortalidad. El estado nutricional fue evaluado a través del déficit de talla para edad, o desnutrición crónica, que tiene alta prevalencia en el Perú $\left({ }^{10}\right)$. Para incrementar la precisión del indicador a nivel departamental, se utlizó promedios de puntaje $\mathrm{Z}$ de talla para edad, en lugar de la proporción por debajo de puntajes $-2 Z\left({ }^{11}\right)$. Se utilizó dos indicadores en los análisis:

- Cambio de talla para edad: promedio departamental de puntaje $\mathrm{Z}$ de talla para edad en el 2000 menos el promedio en 1996 (Endes).

- Promedio de talla para edad: media de los promedios de puntajes $\mathrm{Z}$ de talla para edad de 1996 y del 2000 (Endes).

Para obtener los estimados de mortalidad, fue necesario resolver el problema del subregistro de muertes, que afecta a la mayor parte del país de ingresos bajos y medios. Las encuestas Endes brindan estimados indirectos de la mortalidad de los menores de cinco años en base a las historias de nacimientos. Sin embargo, debido a las restricciones del tamaño muestral, los estimados para el nivel departamental se basan en nacimientos que ocurrieron en los 10 años previos a la encuesta y los resultados de la Endes 2000 se refieren por tanto aproximadamente al año 1995. Tales estimados, entonces, no podrían detectar un impacto reciente de Aiepi. Utilizamos dos métodos alternativos para estimar 
la mortalidad de los niños menores de cinco años en nuestros análisis.

El primer método fue desarrollado por el Centro Latino-Americano de Demografia (Celade) y ampliamente adoptado por la OPS $\left.{ }^{12}\right)$. Para cada departamento del Perú, se identificó una tabla de vida modelo de Celade, que se adecuara a la distribución etárea de la población y de las muertes registradas para cada año, entre 1996 y el 2000. Las tasas de mortalidad correspondientes a estas tablas de vida modelo fueron asignadas luego a cada departamento en cada año particular. Estos resultados fueron compilados por la OPS y expresados como muertes por 100000 niños menores de cinco años. Para permitir las comparaciones con la razón de mortalidad de niños más comunmente usada (U5MR, o muertes de menores de cinco años divididas por los nacimientos ocurridos en el mismo periodo), se multiplicó los estimados de la OPS por 4,7 , en base al nivel de mortalidad actual del Perú.

El segundo método fue utilizar las razones de mortalidad proporcional de la niñez (U5M proporcional) o la proporción de muertes registradas en menores de cinco años sobre todas las muertes registradas. Si bien se sabe que el registro de mortalidad es incompleto, es muy probable que el uso de muertes registradas tanto en el numerador como en el denominador reduzca los sesgos. Se utilizó cuatro indicadores en los análisis:

- U5MR promedio en el periodo 1996-2000 (OPS).

- Reducción acumulada en U5MR en el periodo 1996-2000 (OPS); se calculó la pendiente anual de reducción para cada departamento construyendo una línea de regresión de los niveles de U5MR en años calendario; la reducción acumulada de cinco años fue obtenida de las pendientes anuales.

- U5M proporcional promedio en el periodo 1996-2000 (OEI/Minsa).

- Reducción acumulada en la U5M proporcional en el periodo 1996-2000 (OEI/
Minsa), calculada como se describió anteriormente para la U5MR.

Como ya se indicó, los estimados indirectos de U5MR en base a la Endes 2000 se refieren aproximadamente a 1995 y por tanto son inadecuados para evaluar el impacto de Aiepi. Sin embargo, estos estimados fueron utilizados en nuestros análisis en un intento por validar los estimados de mortalidad descritos arriba. El indicador utilizado fue:

- Estimado indirecto de mortalidad de la niñez, circa 1995 (Endes).

La información sobre diversos factores contextuales fue obtenida a partir de las encuestas nacionales de hogar anuales $\left({ }^{6}\right)$. Aquellos incluidos en nuestros análisis se enumeran a continuación.

- Educación de la mujer: promedio de años de escolaridad para mujeres entre 15 y 44 años de edad, periodo1996-2000.

- Ingreso familiar: ingreso familiar mensual promedio corregido para inflación en los tres meses previos a la encuesta, periodo1996-2000.

- Provisión de agua: porcentaje de hogares con agua de caño dentro de la vivienda, promedio del periodo 1996-2000.

- Desagüe: porcentaje de viviendas conectadas a red pública de alcantarillado, promedio del periodo 1996-2000.

- Electricidad: porcentaje de viviendas con electricidad, promedio del periodo 1996-2000.

Además, se incluyó variables departamentales relacionadas al desarrollo humano (Programa de las Naciones Unidas para el Desarrollo) e indicadores de pobreza (Instituto de Estadística e Informática), las mismas que se enumera a continuación. Se disponía de varios indicadores de pobreza y seleccionamos el que mostró la correlación más fuerte con la mortalidad de la niñez.

- Indice de desarrollo humano, 2000.

- Línea de pobreza: porcentaje de familias que viven bajo la línea de pobreza extrema, 2001. 
También, se obtuvo información sobre la presencia de programas o proyectos de salud del niño diferentes de Aiepi, tanto a nivel departamental como nacional. Se identificó los siguientes proyectos de escala grande y activos durante el periodo 1996-2000: Proyecto Usaid/ Ministerio de Salud "Proyecto 2000", ADRA (Adventist Development and Relief Agency International), y Proyecto Wiñay (Cáritas del Perú). Se identificó otros proyectos en menor escala, pero debido a su baja cobertura no fueron incluidos en los análisis. Debido a que no se dispuso de información precisa sobre los indicadores de cobertura, creamos variables categóricas para estos tres proyectos, indicando si estaban presentes o no en cada departamento.

Los análisis estadísticos incluyeron el uso de los coeficientes de correlación bivariada de Pearson, para evaluar las asociaciones entre la implementación de Aiepi y los indicadores de utilización, cobertura, mortalidad y nutrición. Posteriormente, se calculó coeficientes de correlación parcial para las mismas asociaciones, controlando los efectos potencialmente confusores de los factores contextuales.

\section{RESULTADOS}

Esta sección está organizada como sigue. Primero, se presenta un análisis descriptivo de todas las variables y un reporte de las tendencias temporales. Continúa luego una serie de análisis para validar las variables de mortalidad y talla para edad, correlacionando estas variables con algunos determinantes bien establecidos. Luego, se examina el efecto posible de la capacitación en Aiepi en los indicadores de utilización y cobertura y su impacto sobre mortalidad y talla para edad. Finalmente, se muestra las asociaciones entre indicadores intermedios (utilización y cobertura) y los indicadores de impacto.

Los promedios, las desviaciones estándar y el rango de las variables estudiadas se incluye en la Tabla 1. Entre los 24 departamentos, la cobertura de capacitación en Aiepi clínico estuvo entre $0 \%$ y $66,9 \%$ de los médicos y enfermeras del Minsa. Cuatro departamentos tenían una cobertura de capacitación mayor de $40 \%$. La cobertura de capacitación en Aiepi comunitario varió entre 0 y 41,7 por 10000 niños.

Tabla 1. Estadísticas descriptivas de variables utilizadas en los análisis ( $\mathrm{n}=24$ departamentos).

\begin{tabular}{lrrrr}
\hline Variable & Promedio & Mínimo & $\begin{array}{r}\text { Máximo } \\
\text { Desviación } \\
\text { estándar }\end{array}$ \\
\hline Capacitación en Aiepi clínico (\%) & 20,0 & 0,0 & 66,9 & 17,3 \\
Capacitación en Aiepi comunitario (por 10,000 niños) & 11,3 & 0,0 & 41,7 & 11,0 \\
Consultas externas (promedio visitas/año) & 4,8 & 1,4 & 9,2 & 1,8 \\
Cobertura de vacunas (\%) & 64,2 & 45,4 & 89,1 & 11,9 \\
Promedio de talla para edad (puntaje z) & $-1,2$ & $-2,1$ & $-0,3$ & 0,4 \\
Cambio de talla para edad (puntaje z) & 0,0 & $-0,9$ & 0,6 & 0,3 \\
Promedio de U5MR (por mil nacidos) & 62,0 & 30,7 & 91,1 & 16,8 \\
Reducción acumulada de U5MR 1996-2000 (\%) & 32,5 & 20,3 & 48,5 & 6,7 \\
U5M proporcional promedio (\%) & 18,3 & 7,3 & 35,0 & 6,9 \\
Reducción acumulada promedio de U5M (\%) & 40,3 & 21,5 & 54,6 & 9,9 \\
U5MR indirecta (por mil) & 65,3 & 27,0 & 108,0 & 23,2 \\
Educación de la mujer (años) & 7,2 & 5,9 & 8,8 & 0,7 \\
Ingreso familiar (soles) & 3221 & 1973 & 6414 & 883 \\
Agua potable a domicilio (\%) & 37,8 & 20,5 & 53,7 & 7,3 \\
Desagüe a domicilio (\%) & 28,4 & 12,8 & 52,6 & 8,7 \\
Electricidad a domicilio (\%) & 42,2 & 32,1 & 62,1 & 6,9 \\
\hline Pobreza extrema (\%) & 31,5 & 3,1 & 74,4 & 1,8 \\
\hline Índice de desarrollo humano & 0,58 & 0,46 & 0,75 & 0,07 \\
\hline
\end{tabular}


Tabla 2. Correlaciones entre variables socioeconómicas y ambientales con niveles de mortalidad y desnutrición crónica, valores promedio para 1996-2000.

\begin{tabular}{lcccc}
\hline Variable explicativa & \multicolumn{3}{c}{ Coeficientes de correlación y valores de $p$} \\
\cline { 2 - 5 } & U5MR Promedio & $\begin{array}{c}\text { U5M proporcional } \\
\text { promedio }\end{array}$ & $\begin{array}{c}\text { U5MR indirecta, } \\
\text { circa 1995 }\end{array}$ & $\begin{array}{c}\text { Promedio de talla } \\
\text { para edad puntaje Z }\end{array}$ \\
\hline Educación de la mujer & $-0,714(<0,001)$ & $-0,577(0,003)$ & $-0,709(<0,001)$ & $0,754(<0,001)$ \\
Ingreso familiar & $-0,690(<0,001)$ & $-0,570(0,004)$ & $-0,626(0,001)$ & $0,686(<0,001)$ \\
Agua potable & $-0,709(<0,001)$ & $-0,518(0,009)$ & $-0,644(0,001)$ & $0,614(0,001)$ \\
Desagüe & $-0,680(<0,001)$ & $-0,652(0,001)$ & $-0,558(0,005)$ & $0,647(0,001)$ \\
Electricidad & $-0,606(0,002)$ & $-0,342(0,1)$ & $-0,551(0,005)$ & $0,639(0,001)$ \\
Índice de desarrollo humano & $-0,861(<0,001)$ & $-0,654(0,001)$ & $-0,848(<0,001)$ & $-0,829(<0,001)$ \\
Línea de pobreza & $0,855(<0,001)$ & $0,661(<0,001)$ & $0,926(<0,001)$ & $0,852(<0,001)$ \\
U5MR & 1 & $0,764(<0,001)$ & $0,898(<0,001)$ & $-0,811(<0,001)$ \\
U5M proporcional & - & 1 & $0,640(0,001)$ & $-0,522(0,009)$ \\
U5MR indirecta & - & - & 1 & $-0,838(<0,001)$ \\
\hline
\end{tabular}

La utilización de servicios de consulta externa por niños menores de cinco años fue alta, con un promedio de 4,8 visitas por niño/año, y dos tercios de todos los niños tenían todas las vacunas básicas registradas en sus carnés de salud.

En términos de desnutrición crónica, el promedio global de talla para edad fue igual a 1,2 puntajes $\mathrm{z}$, variando entre $-2,1$ y $-0,3$, indicando por tanto un fracaso de crecimiento sustancial. Se evaluó las tendencias en el estado nutricional a través del cambio en los puntajes z para los años 1996 y 2000. Ocho departamentos mostraron cambios negativos en talla para edad (siendo el mayor un puntaje $\mathrm{z}$ de -0,94) y 16 mostraron cambios positivos (siendo el más alto un puntaje $\mathrm{z}$ de 0,64 ). Por tanto, la talla promedio aumentó en la mayor parte de los departamentos.

El promedio global de la U5MR para el periodo 1996-2000 fue 62,0 (rango 36,7 a 91,1). La reducción acumulada promedio en el periodo fue $32,5 \%$. La U5M proporcional varió de $9 \%$ a $41 \%$ en 1996 y de $5 \%$ a $32 \%$ en 2000 (datos no mostrados). El nivel promedio para el periodo 1996-2000 fue 18,3\% (rango7,3 a 35,0\%). La reducción acumulada promedio para el periodo fue $40,3 \%$ respecto del nivel de 1996 . Solamente un departamento -Huancavelica- mostró un incremento en la mortalidad proporcional. Para el Perú, globalmente la mortalidad proporcional de los menores de cinco años cayó de 18,6\% en
1996 a 11,9\% en 2000. Por tanto, la mortalidad como el estado nutricional mejoraron en el tiempo en la mayoría de departamentos. Dado el modo en que se expresa estas variables, uno esperaría que las mejoras en la atención de salud muestren asociaciones positivas con la reducción de la mortalidad y con el cambio en talla para edad (esto es, que los niños sean de mayor estatura).

Se hizo el intento de validar las estadísticas oficiales sobre mortalidad proporcionada de los menores de cinco años. Endes provee estimados indirectos de mortalidad de la niñez a nivel departamental. Si bien los estimados basados en Endes son afectados por la variabilidad del muestreo y se refieren a una fecha que es aproximadamente cinco años antes de la encuesta, no son afectados por el subregistro, puesto que se basan en las respuestas de las madres. La correlación entre U5MRs en 1996-2000 y U5MRs indirecta (circa 1995) fue igual a $0,90(p<0,001)$ y la correlación entre la última y la U5M proporcional en 1996-2000 fue 0,64 $(p=0,001)$.

También, se puede evaluar la validez de los índices de mortalidad y desnutrición verificando si guardan una buena correlación con determinantes bien conocidos, tales como variables socioeconómicas y ambientales. La Tabla 2 muestra que todas las correlaciones se encuentran en la dirección esperada. Los departamentos con mejores condiciones 
socioeconómicas y ambientales tuvieron menores indicadores de mortalidad y mayor talla para edad. Excepción hecha de la asociación entre electricidad en los domicilios y U5M proporcional, todas las otras correlaciones fueron significativas y muchas realmente fuertes. La Tabla 2 muestra también que las correlaciones entre mortalidad y talla para edad fueron significativas y estuvieron en la dirección esperada.

El modelo de impacto de Aiepi predecía que una de las vías por las que la capacitación en Aiepi podría reducir la mortalidad era a través de un incremento en la utilización de los establecimientos de salud y de la cobertura de intervenciones preventivas, tales como las inmunizaciones. Sin embargo, la correlación entre cobertura de capacitación en Aiepi, consultas ambulatorias y cobertura de vacunas fue débil y no significativa (Tabla 3 ).

Igualmente, no hubo asociaciones significativas entre cobertura de capacitación clínica y los indicadores de mortalidad o nutrición (Tabla 3 y Figuras 2 y 3).

La capacitación de los agentes comunitarios de salud en Aiepi no estuvo asociada con la reducción en la mortalidad, pero hubo una asociación significativa con el cambio en talla para edad en la dirección esperada (Tabla 3 y Figura 4).

Los análisis de tendencias temporales en mortalidad fueron repetidos, tanto para U5MR como para U5M proporcional, usando como la variable de desenlace la razón de los niveles de mortalidad en 1999-2000 sobre los niveles 1996-
1997. Las correlaciones con las variables explicativas fueron virtualmente las mismas que las mostradas en la Tabla 3.

La Tabla 4 muestra la matriz de correlaciones parciales entre capacitación en Aiepi, variables de utilización, cobertura, mortalidad y nutrición, controlando los factores contextuales. Los resultados anteriores permanecieron prácticamente sin cambios, luego de hacer el ajuste correspondiente de estos factores.

El modelo de impacto de Aiepi (Figura 1) especifica que, para que Aiepi tenga un impacto en la mortalidad y el estado nutricional, tendrían que ocurrir varios cambios intermedios. Cuando se exploró dichos cambios, no se encontró asociación alguna entre atenciones de consulta externa y la cobertura de vacunaciones $(r=-0,104$; $p=0,67)$. Igualmente, de acuerdo al modelo de impacto, uno esperaría correlaciones negativas entre variables de utilización/cobertura con mortalidad proporcional y correlaciones positivas con el cambio en talla para edad. Sin embargo, tal como lo muestran las Tablas 3 y 4, la atención de los menores de cinco años no tuvo efectos y la asociación entre cobertura de vacunas y cambio de talla para edad que se observó en los análisis crudos desapareció luego del ajuste.

\section{DISCUSIÓN}

La EMP en el Perú es el primer intento de documentar la implementación de Aiepi a nivel nacional y de buscar evidencia de un impacto sobre los indicadores de salud y nutrición bajo condiciones de efectividad, es decir de

Tabla 3. Coeficientes de correlación bivariada entre implementación de Aiepi e indicadores de utilización, cobertura e impacto. Valores de $p$ entre paréntesis.

\begin{tabular}{|c|c|c|c|c|c|c|}
\hline & $\begin{array}{c}\text { Capacitación } \\
\text { en Aiepi } \\
\text { comunitario }\end{array}$ & $\begin{array}{c}\text { Consultas } \\
\text { externas }\end{array}$ & $\begin{array}{l}\text { Cobertura } \\
\text { de vacunas }\end{array}$ & $\begin{array}{l}\text { Reducción } \\
\text { en U5MR }\end{array}$ & $\begin{array}{c}\text { Reducción } \\
\text { en U5M } \\
\text { proporcional }\end{array}$ & $\begin{array}{l}\text { Cambio } \\
\text { de talla } \\
\text { para edad }\end{array}$ \\
\hline Capacitación en Aiepi clínico & $0,324(0,12)$ & $-0,234(0,27)$ & $0,015(0,95)$ & $0,048(0,82)$ & $0,060(0,78)$ & $0,222(0,29)$ \\
\hline Capacitación en Aiepi comunitario & 1 & $-0,255(0,23)$ & $0,262(0,21)$ & $-0,162(0,45)$ & $-0,091(0,67)$ & $0,505(0,01)$ \\
\hline Consultas externas & - & 1 & $0,131(0,54)$ & $0,150(0,48)$ & $-0,238(0,26)$ & $0,021(0,92)$ \\
\hline Cobertura de vacunas & - & - & 1 & $-0,052(0,81)$ & $-0,300(0,16)$ & $0,461(0,02)$ \\
\hline
\end{tabular}




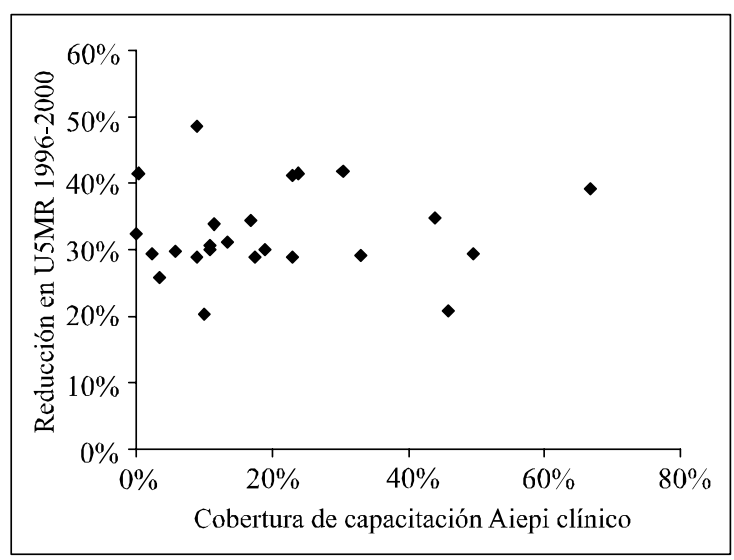

Figura 2. Diagrama de puntos de la reducción en la mortalidad de la niñez de acuerdo a la cobertura acumulada de capacitación en Aiepi clínico (cada punto representa un departamento).

implementación rutinaria. Un estudio recientemente publicado sobre Tanzania sugiere que un Aiepi fuertemente implementado llevó a una reducción de la mortalidad y a una mejora en la talla para la edad $\left({ }^{13}\right)$, en distritos en los que se había implementado previamente con éxito instrumentos de planificación y presupuestación y donde la utilización de los servicios de salud por los niños era alta. Otro estudio reciente mostró que la capacitación en Aiepi clínico y el soporte de los sistemas de salud llevaron a una mejora en la búsqueda de atención y en la utilización de los servicios de salud en Bangladesh $\left({ }^{14}\right)$. El último es un estudio aleatorio cuyo objetivo era evaluar el impacto de Aiepi bajo condiciones controladas de eficacia. Es importante enfatizar que ambos estudios fueron realizados en áreas donde se había producido un fortalecimiento sustancial de los establecimientos de salud, y donde las tasas de mortalidad eran sustancialmente mayores que en el Perú.

Los estudios aleatorios de eficacia, sin embargo, pueden no ser factibles o éticos para evaluar el impacto en gran escala de intervenciones de salud pública $\left({ }^{15}\right)$. Igualmente, las cadenas causales entre el desenlace y las intervenciones de salud pública son usualmente complejas y están sujetas a la modificación de efectos por factores contextuales $\left({ }^{15}\right)$. Un enfoque de plausibilidad tal como el empleado en el

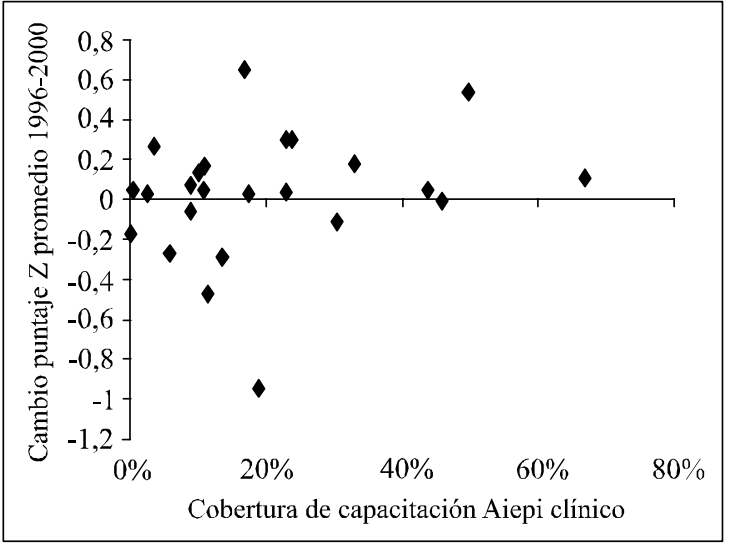

Figura 3. Diagrama de puntos de cambio en el puntaje promedio $\mathrm{Z}$ de talla para edad de acuerdo a la cobertura acumulada de capacitación en Aiepi clínico (cada punto representa un departamento).

presente estudio se constituye por tanto en un complemento útil a los estudios de eficacia. Una consideración cuidadosa de las variables contextuales en el análisis de las asociaciones potenciales es clave para mejorar la confiabilidad de los resultados.

Este estudio estuvo basado en datos secundarios de diversas fuentes, sistemas de información de salud y resultados de encuestas. Se prestó particular interés a la verificación de la calidad y validez de estos datos, especialmente

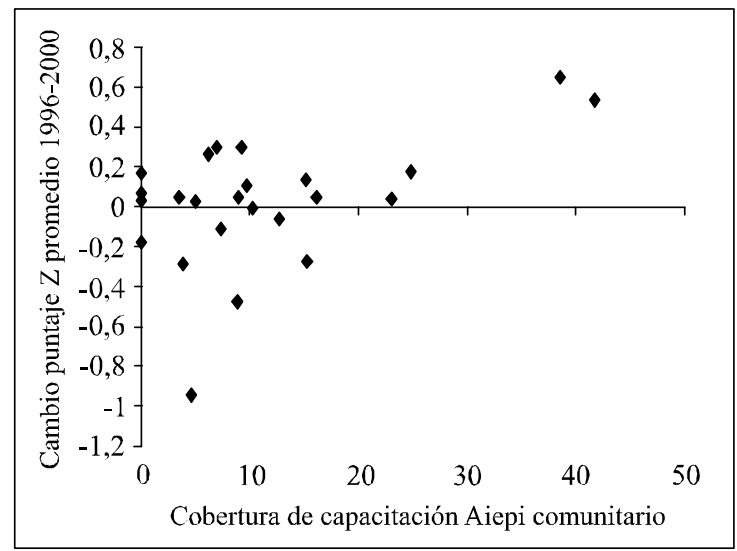

Figura 4. Diagrama de puntos de cambio en el puntaje promedio $\mathrm{Z}$ de talla para edad de acuerdo a la cobertura acumulada de capacitación en Aiepi comunitario expresada en trabajadores de salud por 10000 menores de cinco años (cada punto representa un departamento). 
Tabla 4. Coeficientes de correlación parcial entre implementación de AIEPI e indicadores de utilización, cobertura e impacto, con ajuste de factores contextuales (ingresos, educación, agua, desagüe, electricidad y presencia de otros proyectos de supervivencia de la niñez). Valores de $p$ entre paréntesis.

\begin{tabular}{lcccccc}
\hline & $\begin{array}{c}\text { Capacitación } \\
\text { en Aiepi } \\
\text { comunitario }\end{array}$ & $\begin{array}{c}\text { Consultas } \\
\text { externas }\end{array}$ & $\begin{array}{c}\text { Cobertura } \\
\text { de vacunas }\end{array}$ & $\begin{array}{c}\text { Reducción } \\
\text { en U5MR }\end{array}$ & $\begin{array}{c}\text { Reducción } \\
\text { en U5M } \\
\text { proporcional }\end{array}$ & $\begin{array}{c}\text { Cambio } \\
\text { de talla } \\
\text { para edad }\end{array}$ \\
\hline Capacitación en Aiepi clínico & $0,401(0,15)$ & $-0,414(0,14)$ & $0,258(0,37)$ & $-0,199(0,50)$ & $0,221(0,45)$ & $0,326(0,26)$ \\
Capacitación en Aiepi comunitario & 1 & $0,153(0,60)$ & $0,456(0,10)$ & $0,127(0,67)$ & $0,096(0,75)$ & $0,594(0,03)$ \\
Consultas externas & - & 1 & $0,053(0,86)$ & $0,139(0,64)$ & $-0,137(0,64)$ & $-0,023(0,94)$ \\
Cobertura de vacunas & - & - & 1 & $0,392(0,17)$ & $-0,048(0,87)$ & $0,211(0,47)$ \\
\hline
\end{tabular}

para los estimados de mortalidad, que, empezando por las muertes registradas, hacen uso de técnicas demográficas para corregir el sub-registro $\left({ }^{12}\right)$. Es importante subrayar que los niveles resultantes de mortalidad de la niñez estuvieron muy fuertemente correlacionados con indicadores de pobreza y con índices nutricionales estimados a través de encuestas poblacionales, sugiriendo que los datos de mortalidad son confiables.

La evaluación fue diseñada sobre la base del modelo de impacto mostrado en la Figura 1. El modelo especifica que la introducción y la planificación constituyen el primer paso necesario en el camino a un impacto mensurable de Aiepi. Se esperaba que una implementación exitosa llevara a una mejor disponibilidad de medicamentos, a una mejor supervisión y a otros cambios positivos en el sistema de salud, así como a una mayor capacitación de los trabajadores de salud y de las visitas de seguimiento. Las actividades a nivel comunitario debían tener lugar en las mismas áreas geográficas y al mismo tiempo. Problemas con la disponibilidad de medicamentos o una capacitación y supervisión adecuada pueden impedir mejoras en el cumplimiento a nivel del hogar, en la búsqueda de atención y en la utilización de los servicios de salud. Por otra parte, si la calidad de atención en los establecimientos de salud es inadecuada o si las prácticas de búsqueda de atención, utilización y cumplimiento no mejoran, no podría esperarse que la salud y la nutrición del niño mejoren.

Aiepi fue introducida en el Perú a fines de 1996 y fue seguida de una fase de expansión, que continuó hasta 1999. Ésta fue seguida por una disminución en la velocidad de capacitación en Aiepi $\left({ }^{5}\right)$. La cobertura de capacitación lograda hacia fines del 2001 fue relativamente baja; la cobertura nacional fue $10,2 \%$, pero cuatro departamentos estuvieron por encima de $40 \%$. Para propósitos de monitoreo, un centro o puesto de salud es considerado como establecimiento Aiepi si por lo menos $60 \%$ de sus trabajadores de salud están capacitados en Aiepi $\left({ }^{16}\right)$. En la EMP, implementación plena de Aiepi fue definida como aquella en la que por lo menos $80 \%$ de los establecimientos de salud en una región dada alcanzó esta meta. La misma requiere que $48 \%$ (60\% de $80 \%$ ) o más de todos los trabajadores de salud en el departamento estén capacitados en Aiepi, un nivel que solamente fue alcanzado por dos departamentos en el Perú.

Adicionalmente, el soporte de los sistemas de salud para Aiepi en el Perú fue variable. Por otra parte, la disponibilidad de medicamentos y equipos fue buena en general y la utilización de los servicios de salud fue alta (promedio de más de cuatro visitas por niño menor de cinco años, por año). Las actividades de supervisión en cambio fueron insuficientes, Aiepi no estuvo adecuadamente institucionalizada o presupuestada ni a nivel nacional ni a nivel departamental y la rotación del personal capacitado fue muy elevada $\left({ }^{5}\right)$.

Tanto la mortalidad como el estado nutricional de los niños mejoraron en la mayor parte del Perú, de 1996 al 2000. Se esperaría que los avances en la atención de salud, incluyendo la 
implementación de Aiepi, resulten en mayores reducciones en la mortalidad de la niñez y en cambios positivos de talla para edad. Sin embargo, no se constató tales asociaciones en el Perú. Una explicación posible es que la cobertura de capacitación en la mayor parte de los departamentos fue muy baja como para resultar en un impacto cuantificable. Esto es probablemente cierto, pero debe indicarse que incluso en los cuatro departamentos con una cobertura clínica mayor de $40 \%$ no hubo evidencia de mayores mejoras en la salud del niño que en los restantes 20 departamentos. Estos resultados no cambiaron luego de controlar las variables contextuales.

Una limitación importante de la implementación de Aiepi en el Perú fue la falta de coordinación entre la capacitación de los trabajadores clínicos y la de los agentes comunitarios de salud, que a menudo tuvo lugar en áreas geográficas diferentes $\left(^{5}\right)$. El último componente empezó en 1997 y fue expandido rápidamente -para el 2000, cuatro departamentos tenían más de 20 agentes comunitarios capacitados por cada 10,000 niños menores de cinco años-. La cobertura de capacitación en Aiepi comunitario no estuvo asociada con los indicadores de mortalidad, pero hubo una correlación positiva y significativa con una mejor talla para la edad, la que se mantuvo luego de controlar los factores contextuales. Es difícil afirmar si ésta es o no una asociación causal, pues las otras correlaciones entre capacitación en Aiepi comunitario y variables intermedias o de desenlace no fueron significativas. Por tanto, dado que se evaluó un número grande de correlaciones, no puede descartarse que el hallazgo sea casual. Se necesita mayor investigación sobre este tema.

La falta de correlación entre la implementación de Aiepi, medida por la cobertura de capacitación, y los indicadores de utilización de servicios de salud, cobertura e impacto deben ser interpretados a la luz de los estudios mencionados en Tanzania y Bangladesh. En ambos estudios, se demostró efectos positivos, pero la capacitación en Aiepi clínico sólo era parte de una estrategia más amplia que incluía un fuerte componente de fortalecimiento de los sistemas de salud. La provisión de medicamentos, vacunas y equipos fue adecuada, las actividades de supervisión estaban presentes y la tasa de rotación del personal fue baja. Aiepi estaba fuertemente institucionalizada a nivel distrital, y la cobertura de capacitación entre los trabajadroes clínicos era cercana al 100\% en los ámbitos e estudio, tanto en Tanzania como en Bangladesh. Estos resultados, por lo tanto, no son necesariamente contradictorios con los resultados del estudio peruano, que refleja una situación cotidiana, en circunstancias reales, en las que Aiepi no fue plenamente implementada.

Adicionalmente, los niveles de mortalidad basal fueron considerablemente menores en el Perú que en Tanzania y Bangladesh, donde una gran proporción de muertes se debe a las cinco condiciones (diarrea, neumonía, malaria, sarampión y malnutrición) a las que está dirigida Aiepi. La estructura de mortalidad del Perú puede ser menos susceptible al impacto de Aiepi, si bien la diarrea y la neumonía constituyen todavía una cuarta parte de todas las muertes en menores de cinco años. También la prevalencia de desnutrición crónica en el Perú es todavía muy alta y existe ciertamente bastante lugar para un impacto de Aiepi, particularmente en las áreas pobres del país.

El contraste entre los resultados de los tres estudios revela la importancia de llevar a cabo evaluaciones de plausibilidad en gran escala de intervenciones que están siendo expandidas, así como estudios de menor escala, tales como los de Tanzania y Bangladesh, en los que la intervención es llevada a cabo de una manera más intensa y controlada. Las lecciones aprendidas acerca de las barreras para expandir Aiepi en el Perú $\left(^{5}\right)$ pueden ser de beneficio para otros países que están implementando la estrategia, así como otras estrategias -tales como la estrategia ' 3 por 5 ' $\left({ }^{17}\right)$, que son grandemente dependientes de sistemas de salud funcionalmente adecuados. Las agencias financiadoras de investigación deben también prestar especial atención a la necesidad de apoyar evaluaciones de efectividad de gran escala $\left({ }^{18}\right)$. 


\section{AGRADECIMIENTOS}

Este trabajo es parte de la Evaluación Multi-País de la Efectividad, Costo e Impacto de Aiepi (EMP), coordinada por el Departamento de Salud del Niño y el Adolescente de la Organización Mundial de la Salud, y financiada por la Fundación Bill y Melinda Gates y por la Agencia para el Desarrollo Internacional de los Estados Unidos de Norte América. El Instituto de Estadística e Informática del Perú (INEI) brindó las bases de datos de Endes -Encuesta Nacional Demográfica y de Salud Familiar (1996 and 2000), y Enaho -Encuesta Nacional de Hogares (1996-2000). El Dr. Guillermo Guibovich de la OPS, Lima, Perú, brindó varios datos secundarios. El personal del Ministerio de Salud fue de gran ayuda en la provisión de datos adicionales a nivel nacional y regional.

\section{REFERENCIAS BIBLIOGRÁFICAS}

1. Black RE, Morris SS, Bryce J. Where and why are 10 million children dying every year? The Lancet. 2003;361:2226-34.

2. Department of Child and Adolescent Health and Development, World Health Organization. Estimated coverage of IMCI training [map]. Geneva, Switzerland: WHO; 2004 [citado 2005-01-20]. Disponible en: http:// www. who.int/child-adolescent-health/OVERVIEW/ CHILD HEALTH/map12 02.jpg

3. Hill Z, Kirkwood B, Edmond K. 2002. Family and community practices that promote child survival, growth and development: a review of the evidence [monografía en la Internet]. Geneva, Switzerland: WHO; 2002 [citado 200501-20]. Disponible en: http://www.who.int/child-adolescenthealth/New Publications/CHILD HEALTH/ ISBN_92_4_159150_1.pdf

4. Bryce J, Victora CG, Habicht JP, Black RE, Vaughan JP. The Multi-Country Evaluation of the Integrated Management of Childhood Illness Strategy. Am J Public Health. 2004;94:406-15.

5. Huicho L, Davila M, Campos M, Drasbek C, Bryce J, Victora CG. Scaling up integrated management of childhood illness to the national level: achievements and challenges in Peru. Health Policy Plan. 2005;20:14-24.

6. Instituto Nacional de Estadística e Informática (INEI). Encuesta Nacional de Hogares: ENAHO, 1996-2000. Lima, Perú: INEI; 2000.

7. Instituto Nacional de Estadística e Informática (INEI). Encuesta Demografica y de Salud Familiar: ENDES III, 1996. Lima, Perú: INEI; 1996.

8. Instituto Nacional de Estadística e Informática (INEI). Encuesta Demografica y de Salud Familiar: ENDES IV, 2000. Lima, Perú: INEI; 2000.
9. Oficina General de Estadística e Informática (OGEI). Información de Salud 1996-2000: Ministerio de Salud del Perú. Lima, Perú: OGEI; 2004.

10. Department of Nutrition for Health and Development, World Health Organization [página web en la Internet]. Geneva, Switzerland: WHO; 2004 [citado 2005-01-20]. WHO Global database on child growth and malnutrition; [apróximadamente 4 pantallas]. Disponible en: http:// www. who.int/nutgrowthdb/

11. Expert Commmittee on Nutrition, World Health Organization. Physical status: uses and interpretation of anthropometry. Geneva, Switzerland: WHO; 1995. (WHO Technical Report Series, No. 854).

12. Pan American Health Organization. Technical notes [monografía en la Internet]. In: Health statistics from the Americas. Washington, D.C.: PAHO; 2003. p. 7-9 [citado 2005-01-20]. Disponible en: http:// miranda.ingentaselect.com $/ \mathrm{vl}=3429960 / \mathrm{cl}=25 / \mathrm{nw}=1 /$ $\mathrm{fm}=\mathrm{docpdf} / \mathrm{rpsv} / \mathrm{bk} / \mathrm{paho} / 9275115915 / \mathrm{v} 1 \mathrm{n} 1 / \mathrm{s} 3 / \mathrm{p} 1$

13. Schellenberg JRMA, Adam T, Mshinda H, Masanja H, Kabadi G, Mukasa O, et al. Effectiveness and cost of facilitybased Integrated Management of Childhood Illness (IMCI) in Tanzania. Lancet. 2004;364:1583-94.

14. El Arifeen S, Blum LS, Hoque DM, Chowdhury EK, Khan $\mathrm{R}$, Black RE, et al. Integrated management of childhood illness (IMCI) in Bangladesh: early findings from a clusterrandomised study. Lancet. 2004;364:1595-602.

15. Victora CG, Habicht JP, Bryce J. Evidence-based public health: moving beyond randomized controlled trials. Am J Public Health. 2004;94:400-5.

16. The Interagency Working Group on IMCI Monitoring and Evaluation (BASICS, CDC, UNICEF, USAID, WHO). Indicators for IMCI at first-level facilities and households [monografía en la Internet]. Geneva, Switzerland: WHO; 2001 [citado 2004-11-04]. Disponible en: http:// www.who.int/child-adolescent-health/New_Publications/ CHILD HEALTH/indicators for IMCI.htm

17. World Health Organization. Treating 3 million by 2005. Making it happen [monografía en la Internet]. Geneva, Switzerland: WHO; 2003 [citado 2005-01-20]. Disponible en: http://www.who.int/3by5/publications/documents/ isbn9241591129/en/

18. Mexico, 2004: Global health needs a new research agenda [editorial]. Lancet 2004;364:1555-6.

Manuscrito recibido el 07 de diciembre de 2005 y aceptado para publicación el 20 diciembre de 2005.

Correspondencia: Dr. Luis Huicho

Batallón Libres de Trujillo 227, Surco

Lima 33, Perú

Correo-e:Ihuicho@viabcp.com 